Shoval, S., Ulrich, I., and Borenstein, J., "Computerized Obstacle Avoidance Systems for the Blind and Visually Impaired.” Invited chapter in "Intelligent Systems and Technologies in Rehabilitation Engineering." Editors:

Teodorescu, H.N.L. and Jain, L.C., CRC Press, ISBN/ISSN: 0849301408, Publication Date: 12/26/00, pp. 414-448.

\title{
Computerized Obstacle Avoidance Systems for the Blind and Visually Impaired
}

\author{
by
}

\author{
Dr. Shraga Shoval* Mr. Iwan Ulrich** and Dr. Johann Borenstein***
}

* Faculty of Industrial Engineering and Management - Technion, Israel

** Robotics Institute, Carnegie Mellon University, Pittsburgh, Pennsylvania, USA

*** Department of Mechanical Engineering and Applied Mechanics, The University of Michigan, Ann Arbor, Michigan, USA.

\begin{abstract}
This chapter gives an overview of existing devices for the guidance of visually impaired pedestrians and discusses the properties of the white cane and of conventional electronic travel aids. Also described are the disadvantages of using a standard mobile robot for this purpose. Next follows a description of the NavBelt, a computerized travel aid for the blind that is based on advanced mobile robot obstacle avoidance technology. The NavBelt is worn by the user like a belt and, via a set of stereo earphones, provides acoustic signals that guide the user around obstacles. One limitation of the NavBelt is that it is exceedingly difficult for the user to comprehend the guidance signals in time to allow fast walking.

This problem is effectively overcome by a newer device, called GuideCane. The GuideCane uses the same mobile robotics technology as the NavBelt but it is a wheeled device pushed ahead of the user via an attached cane. When the GuideCane detects an obstacle it steers around it. The user immediately feels this steering action and can follow the GuideCane's new path easily and without any conscious effort. This chapter describes the GuideCane system, including the mechanical, electronic, and software components, followed by a description of the intuitive user-machine interface. The chapter ends with a discussion of the GuideCane's novel information transfer approach and its advantages and disadvantages in practical term.
\end{abstract}

\section{Introduction}

There are about two million visually impaired or blind persons in the United States alone [Jackson, 1995]. Many of these persons use the white cane - the most successful and widely used travel aid for the blind. This purely mechanical device is used to detect obstacles on the ground, uneven surfaces, holes, steps, and other hazards. The inexpensive white cane is so lightweight and small that it can be folded and slipped into a pocket.

The main problem with the white cane is that users must be trained in its use for more than one hundred hours - a substantial "hidden" cost. In addition, this device is rather inconvenient, as it requires the user to actively scan the small area ahead of him $/$ her. In addition, the white cane cannot detect obstacles beyond its reach ( 3 - 6 feet), and therefore the traveler perceives only limited information about the environment. Another drawback of the white cane is that obstacles can be detected only by contact. This can become inconvenient to 
the traveler and the surroundings, for example, when traveling in a crowded street. Travel can even become unsafe through improper use of the white cane. The white cane is also not well suited for detecting potentially dangerous obstacles at head level.

Guide dogs are very capable guides for the blind, but they require extensive training. Fully trained guide dogs cost between $\$ 12,000$ to $\$ 20,000$ [Jackson, 1995], and their useful life is typically on the order of only five years. Furthermore, many blind and visually impaired people are elderly and find it difficult to care appropriately for another living being. As a result, only $1 \%$ of the estimated two million blind and visually impaired people in the U.S. have guide dogs.

\section{Conventional Electronic Travel Aids}

With the development of radar and ultrasonic technologies, a new series of devices, known as electronic travel aids (ETAs) was developed for blind travelers during the past 30 years. In terms of operational principles, most ETAs are similar to radar systems: a laser or ultrasonic "beam" is emitted in a certain direction in space; the beam is reflected from objects that it confronts on its way; a matching sensor detects the reflected beam and the distance to the object is calculated according to the time difference between emitting and receiving the beam. Existing ETAs can detect objects in the range of up to 15 feet away from the user, but require continuous scanning of the environment in the desired direction (with the exception of the Binaural Sonic Aid and the Pathsounder, which depend on head or torso movements).

The best known ETA is the C5 Laser Cane [Benjamin et al.,1973], which is based on optical triangulation with three transmitters and three photodiodes as receivers. An UP channel detects obstacles at head-height, the FORWARD channel detects obstacles from the tip of the can forward, (in the range of 1.5-3.5m) and the DOWN channel detects drop-offs in front of the user.

The Mowat Sensor [Pressey, 1977] is another hand-held device that informs the user of the distance to detected objects by means of tactile vibrations, where the frequency of the vibrations is inversely proportional to the distance between the sensor and the object. The Mowat sensor is a secondary aid for use in conjunction with a long cane or a guide dog. The Mowat sensor has been found helpful, and users feel they benefit from it [Shao, 1985].

The Russell Pathsounder [Russell, 1965] is one of the earliest ultrasonic travel aids. Two ultrasonic transducers are mounted on a board that the user wears around the neck, at chest height. This unit provides only three discrete levels of feedback (series of clicks), roughly indicating distances to objects. The Pathsounder does not require active manual scanning of the environment by the user, but torso movement is the only search strategy potential [Mann, 1974].

The Binaural Sonic Aid (Sonicguide) [Kay, 1974] comes in the form of a pair of spectacle frames, with one ultrasonic wide-beam transmitter $\left(55^{\circ}\right.$ cone $)$ mounted between the spectacle lenses and one receiver on each side of the transmitter. Signals from the receivers are shifted and presented separately to the left and right ear. The resulting interaural amplitude difference allows the user to determine the direction of the reflected echo and thus of the obstacle. The distance to an object is encoded in the frequency of the demodulated low-frequency tone, which together with the wearer's head orientation provides clear information about the object's location. As the Sonicguide does not require active manual scanning, it can serve as a secondary device, in conjunction with an additional hand-held device or a guide dog. 
Three fundamental shortcomings can be identified in all ETAs discussed in the foregoing sections:

1. The user must actively scan the environment to detect obstacles (no scanning is needed with the Sonicguide, but that device does not detect obstacles at floor level). This procedure is time-consuming and requires the traveler's constant activity and conscious effort.

2. The traveler must perform additional measurements when an obstacle is detected in order to determine the dimensions and shape of the object. The user must then plan a path around the obstacle. Again, a time-consuming, conscious effort that reduces the walking speed.

3. Another problem with all ETAs based on acoustic feedback is their interference (called masking) with sound cues from the environment, reducing the blind person's ability to hear these essential cues. [Lebedev and Sheiman, 1980; Kay, 1974; Brabyn, 1982].

\section{Mobile Robotics Technologies for the Visually Impaired}

Visually impaired people and mobile robots face several common problems when performing navigation. Hence, it seems only natural to apply mobile robotics technologies to assist the visually impaired. Obstacle avoidance systems (OASs), originally developed for mobile robots, lend themselves well for incorporation in travel aids for the visually impaired. An obstacle avoidance system has to detect obstacles through its sensors and has to plan a path around them. Travel aids equipped with mobile robotics technologies have the potential of overcoming the fundamental shortcomings of existing travel aids, and can thus provide several advantages to the blind traveler.

\subsection{Mobile Robot Obstacle Avoidance Sensors}

Most mobile robots use ultrasonic sensors (also called "sonars" in the remainder of this chapter) to detect obstacles and to measure the distance between the sensor and the obstacle. By far the most widely used ultrasonic sensor in mobile robot applications is the one manufactured by [Polaroid] and shown in Figure 1.

The Polaroid sonar emits a short burst of ultrasound when it is "fired." If an object is located in front of the sensor, then some of the ultrasound waves will be reflected back to the sonar, which switches into a microphone mode immediately after firing. Once the echo from the object is received at the sonar, its associated electronics (see Figure 1) sends an electrical signal to the computer that controls the sonar(s). The computer measures the time that elapsed between firing the sonar and receiving the echo. Because the velocity of ultrasound traveling through air is almost constant, the computer can easily compute the distance between the object and the sonar from the measured time-of-flight. Polaroid sonars have a maximum range of 10 meters $(33 \mathrm{ft}$ ) and an accuracy of about $0.5 \%$ of the distance measured. 
Ultrasound waves propagate from the sonar in a cone-shaped propagation profile, in which the opening angle of the cone is about $30^{\circ}$ (see Figure 2). This property is exploited in most mobile robots by mounting the sonars on a semi-circular ring on the periphery of the robot at 15-degree intervals. This arrangement assures complete coverage of the area in front of the sonars.

One major difficulty in the use of multiple ultrasonic sensors is the fact that these sensors cause mutual interference, called crosstalk. Crosstalk is a phenomenon in which the wave-front emitted by one sonar specularly reflects off smooth surfaces and is subsequently detected by another sonar. In the past, researchers had to employ slow firing schemes to allow each sensor's signal to dissipate before the next sensor was fired.

This problem was resolved by the Error Eliminating Rapid Ultrasonic Firing (EERUF) method, developed at the University of Michigan's Mobile Robotics Lab [Borenstein and Koren, 1995]. EERUF detects and rejects crosstalk so that there is no need to wait for echoes to dissipate. As a result it is possible to fire sonars at a rate of up to $60 \mathrm{~ms}$. (i.e., each sensor fires once every $60 \mathrm{~ms}$ ). This fast firing technique allows faster sensor acquisition, and thus faster travel. The EERUF algorithm also rejects environmental ultrasonic noise, and filters out erroneous readings.

\subsection{Mobile Robot Obstacle Avoidance}

A mobile robot obstacle avoidance system comprises of a set of sensors and, typically, some rather complex computer algorithms that use the sensor data to compute a path that would lead the robot safely around the object. There are as many different obstacle avoidance methods as there are research labs around the world. However, in both the NavBelt and the GuideCane an algorithm known as the Vector Field Histogram (VFH) method is used, which was developed at the University of Michigan's Mobile Robotics Lab. The VFH method is explained in the following section.

\subsubsection{The Vector Field Histogram Method for Obstacle Avoidance}

The Vector Field Histogram (VFH) method builds a local map of its immediate surroundings

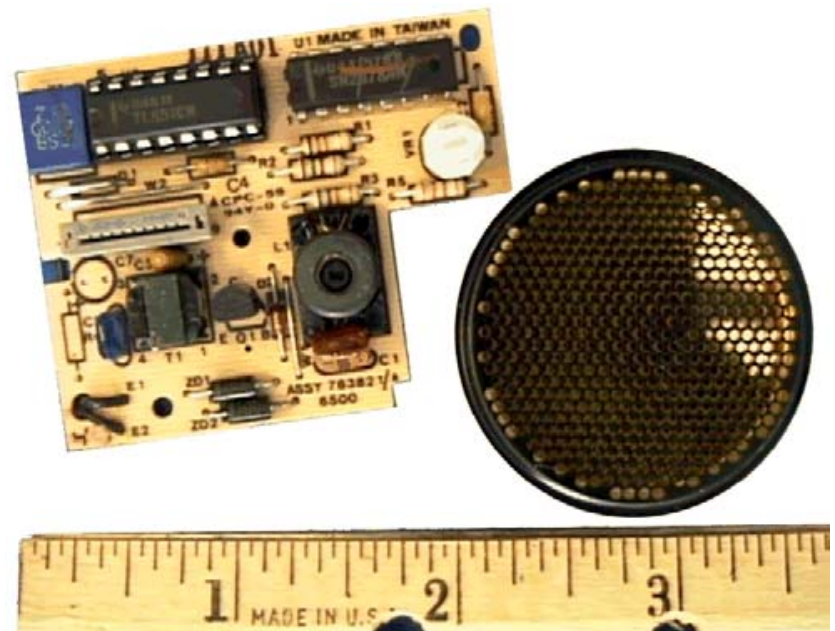

Figure 1: The Polaroid ultrasonic range sensor is widely used for mobile robot obstacle avoidance.

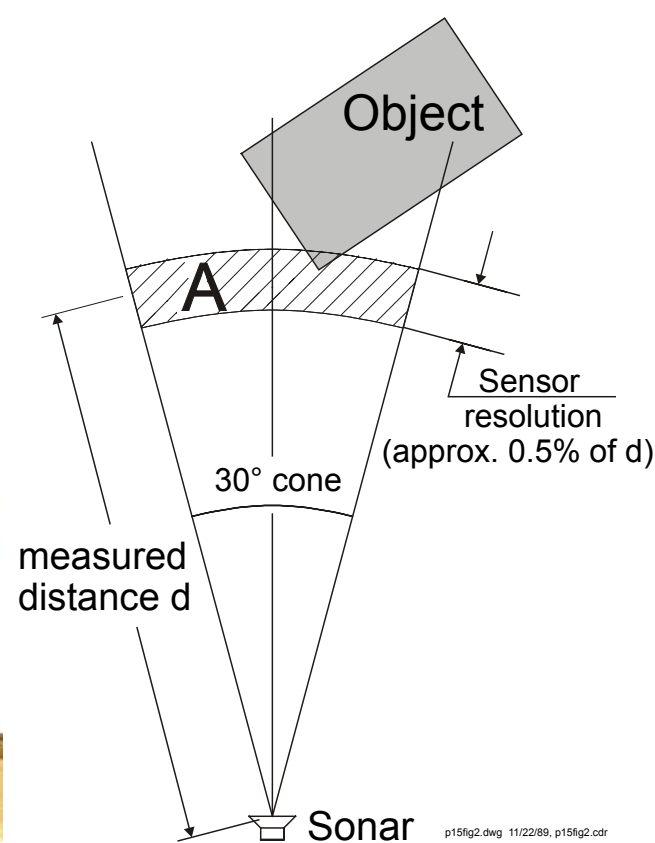

Figure 2: ultrasound propagates from the sonar in a $30^{\circ}$ cone-shaped profile. 
based on the recent sonar data history. This local map allows the robot ${ }^{1}$ to take into account not only the current sonar readings, but also previous readings. This results in significantly better performance than that produced by simpler systems.

In the VFH method the map is represented by a two-dimensional array, called a histogram grid [Borenstein and Koren, 1991], which is based on the earlier certainty grid [Moravec, 1988] and occupancy grid [Elfes, 1989] approaches. Each cell contains a certainty value that indicates the measure of confidence that an obstacle exists within the cell area. This representation is especially suited for sensor fusion, as well as for the accommodation of inaccurate sensor data such as range measurements from ultrasonic sensors. In both the NavBelt and GuideCane a cell size of $10 \times 10 \mathrm{~cm}$ is used.

Figure 3, which was created in an actual experiment, illustrates how a typical experimental environment translates into the histogram grid representation.

The local map is updated through histogramic in-motion mapping (HIMM), a real-time map building method developed by Borenstein and Koren [1991]. HIMM increases the certainty value of only one cell in the histogram grid for each sonar reading, and decreases the certainty values of cells located between the sonar and the incremented cell. While this approach may seem like an oversimplification, a probability distribution is actually obtained by continuous and rapid sampling of each sensor while the robot is moving. In addition, to compensate for the adverse scattering effects caused by in-motion sampling, a growth rate operator (GRO) is added. The GRO increases the value of a cell faster if its immediate neighbors hold high certainty values. As a result, HIMM produces high certainty values for cells that correspond to obstacles and keeps low certainty values for cells that were increased because of misreadings or moving objects. Any range reading is immediately represented in the map and can thus immediately influence the obstacle avoidance output.

1 The term "robot" is used here because the VFH method was originally developed for mobile robots. However, the discussion is true for both the NavBelt and GuideCane, as described later. 
EERUF and HIMM are an effective combination for building good representations of a robot's immediate surroundings based on ultrasonic sensors. The resulting histogram grid is then used by the local obstacle avoidance algorithm as its world model to determine an appropriate instantaneous direction of motion.

Next, the two-dimensional histogram grid is reduced to a one-dimensional polar histogram that is constructed around the robot's momentary location (the mathematical process is beyond the scope of this text and is omitted here). The polar histogram provides an instantaneous 180-degree panoramic view of the immediate environment, in which elevations suggest the presence of obstacles and valleys suggest that the corresponding directions are free of obstacles. The polar histogram has 72 sectors that are each $5^{\circ}$ wide. The numerical values associated with each sector are called "obstacle density" values. High obstacle density values suggest a high likelihood for either a small object nearby or a larger object further away in the direction of that sector. Figure 4 shows the polar histogram
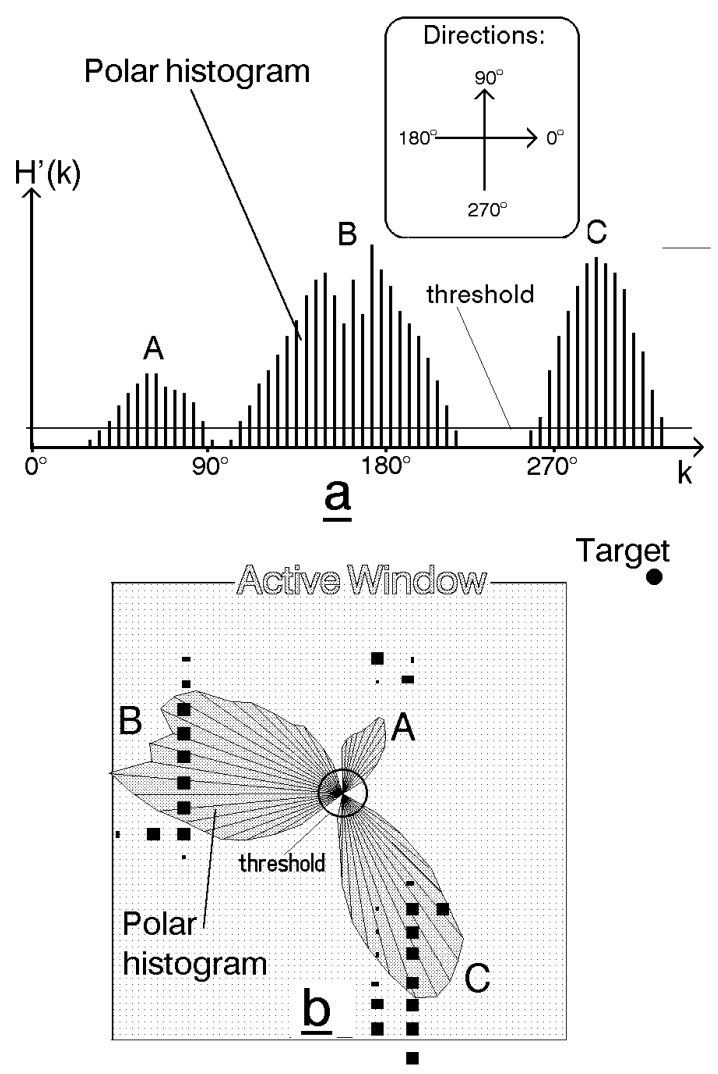

Figure 4: Two ways of visualizing the same polar histogram generated at instant $t$ for the environment of Figure 3.

for the environment of Figure 3 and it was also created from an actual experiment. High obstacle density values are shown as taller bars in the bar chart-type representation of Figure 4a. Note that Figure $4 \mathrm{~b}$ represents the same polar histogram as that of Figure 4a, except that it has been overlaid onto the histogram grid for better illustration.

Although the description in this section is fairly complex, the necessary computations are performed in just a few milliseconds. Indeed, during motion a new polar histogram is recomputed every 10 milliseconds.

It is evident from Figure $4 \mathrm{~b}$ that the polar histogram provides comprehensive information about the environment (with regard to obstacles), yet the amount of numeric information is quite small. Exactly how the information of the polar histogram is used in a robotic device differs from application to application, and will be discussed separately for the NavBelt and the GuideCane in the respective sections below.

\subsubsection{Limitations of Mobile Robots as Guides for the Blind}

In general terms, one could argue that any mobile robot with obstacle avoidance can be used as a guiding device for the blind. However, conventional mobile robots with powered wheels are inherently unsuited to the task of guiding a blind person. Actively driven wheels require motors and thus more powerful batteries, making a standard mobile robot larger and heavier than the NavBelt and the GuideCane. The added weight and size are a considerable inconvenience for a user whenever he/she encounters situations like stairs or raised sidewalks. 
Another problem with powered wheels is that the speed of the robot could make the user feel uncomfortable by either pulling a cautious user forward or by slowing a confident user down unnecessarily. An additional interface would be required so that the user could indicate the desired speed to the robot. However, with both the NavBelt and the GuideCane configuration, the user is in direct control of the speed, allowing for the most intuitive and easiest use possible.

Another concept is to have a visually impaired person sit in a powered semiautonomous wheelchair equipped with sensors and obstacle avoidance technology. The main problem of this approach is that a visually impaired user with healthy legs would unnecessarily be burdened with the additional handicap of limited mobility.

\section{The NavBelt}

For more than 10 years, the University of Michigan Mobile Robotics Laboratory has been active in applying its technologies to travel aids for the visually impaired. The major results are two novel devices: the NavBelt and the GuideCane, whose concepts originated in 1989 and 1995 respectively. Both devices rely on advanced obstacle avoidance systems that were originally developed for conventional mobile robots. This section describes the NavBelt, which overcomes the first two shortcomings of existing travel aids by employing multiple sensors that free the user from scanning the surroundings manually.

\subsection{Concept}

The NavBelt consists of a belt, a small computer worn as a backpack, and an array of ultrasonic sensors. The computer processes the signals arriving from the sensors, applies the obstacle avoidance algorithms, and relays them to the user via stereophonic headphones, using so-called stereo imaging techniques.

The NavBelt system does not use a mobile robot, but it applies mobile robot technology in a portable device. This technology transfer is illustrated in Figure 6. The main difference is that the electrical signals, which originally guided a robot around obstacles, are replaced by acoustic signals aimed at guiding the user around obstacles. However, the computation of an obstacle-free path and the sensing techniques are similar in both applications.

The NavBelt is equipped with an obstacle avoidance system (OAS) that scans the environment with eight sonars simultaneously. The OAS employs a unique real-time signalprocessing algorithm to produce active guidance signals.

The OAS computes the recommended traveling direction according to the user's current position, target location, and the obstacles in the surroundings by using a method called the Vector Field Histogram (VFH) [Borenstein and Koren, 1991]. In the absence of obstacles, the recommended direction is simply the direction toward the target. If, however, obstacles block the user's path, then the OAS computes an alternative path, which safely guides the user around the obstacles. 
The user wears the NavBelt around the waist like a "fanny pack" (see Figure 5 ), and carries a portable computer as a backpack. Eight ultrasonic sensors, each covering a sector of $15^{\circ}$ are mounted on the front pack, providing a total scan sector of $120^{\circ}$. Small stereophonic headphones provide the user with the auditory data. A binaural feedback system (BFS) based on internal time difference (phase difference between the left and right ear) and amplitude difference (volume difference between the two ears) creates a virtual direction (i.e. an impression of directionality of virtual sound sources).

The NavBelt is designed for three basic operational modes, each offering a different type of assistance to the user:

1) Guidance Mode - The acoustic signals actively guide the user around obstacles in pursuit of the target direction. The signals carry information regarding the recommended travel direction as well as
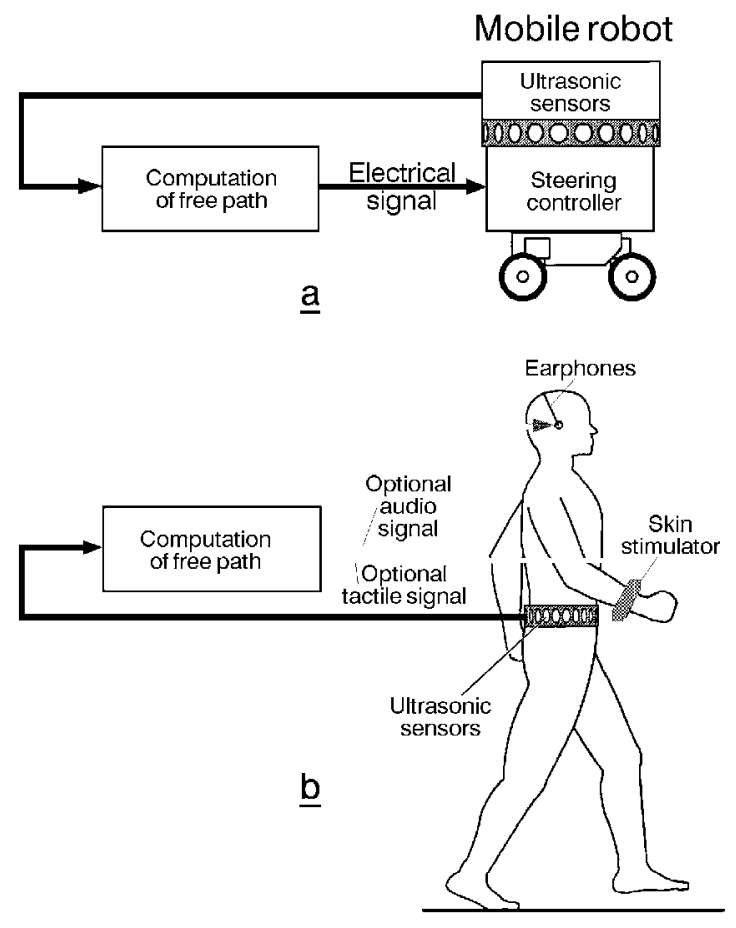

Figure 6: Transferring mobile robotics technology to a portable navigation aid for the blind: The concept of the NavBelt.

\section{- Side Panel: Auditory Displays}

The use of auditory displays in travel aids for the blind has been investigated by several researchers:

- Maure et. al. [1979]. They built an experimental device for testing the effect of various auditory signals. The device consisted of one ultrasonic sensor and a micro computer, which provided eight methods for auditory display. The range readings were processed by the computer, and according to the operational mode (selected manually by the user), auditory signals were transferred to the user. These signals ranged from verbal messages (specifying the distance to an obstacle in inches), to continuous-tone sounds, in which the pitch was proportional or inversely proportional to the distance. Another tested signal was an audible alarm that was activated when the distance to an object became smaller or larger than a specified value (Go-No Go detector).

- Tachi et al., [1983] developed a method to quantitatively compare various auditory display schemes for tracking with an electronic travel aid. The performance of the traveler was evaluated by calculating the transfer function of the human in terms of effective gain and reciprocal time delay. It was found that monaural displays with varying loudness and binaural displays with varying position and loudness are superior to other types of auditory displays. Fish [1976] suggested using a twodimensional coding system, which produces a series of tone bursts representing distances to objects. Although laboratory experiments showed that subjects were able to avoid obstacles using the twodimensional auditory display, the information complexity and size did not allow the traveler to walk at a reasonable speed.

- Ifukube et al. [1991] devised an interesting method for information transfer in a travel aid for the blind, which was based on the principle of echolocation of bats. In this method, frequency modulated ultrasonic waves were transmitted to detect objects, and the reflected waves were picked up by a two-channel receiver. The waves were then converted to acoustic signals with a simple proportional converter. The acoustic signals were presented to the blind traveler binaurally by headphones. Experiments showed that the method was very effective at detecting small objects, but not practical experiments were reported for implementing it in a travel aid.

- Kay's [1974] Binaural Sensory Aid, also known as Sonicguide, relays information to the user by two sound sources to the left and right ears, using special tubes for minimal occlusion of external auditory cues. The interaural differences between the two sound sources provide the user with directional information about objects, as well as the object's shape and even a rough estimate about the surface's texture. 
the speed and proximity to obstacles. The signals consists of a single stereophonic tone, the direction of which determines the travel direction, while the frequency determines the recommended travel speed (higher frequencies for slower speeds). The speed is inversely proportional to the proximity to the nearest object. Using a keyboard (which can eventually be replaced by an acoustic coding system using other input device suitable for blind users), the user inputs the desired target position. The target can be selected as relative coordinates (i.e. $500 \mathrm{ft}$ forward, turn right, etc.), or, when traveling in a known environment and the computer is equipped with a navigation map, the user can specify the target name (i.e. street corners, specific buildings etc). One problem with the Guidance Mode is that it requires knowledge about the user's momentary position at all times. In the current NavBelt prototype there are no sensors that can provide this information. However, developments in positioning method, such as satellite-based systems (GPS) for outdoor environments, may provide solution to this problem. Golledge et al. [1991] developed a navigation aid based on GPS technology, which provides the user with updated information about the topographical features of the surroundings.

2) Image Mode - This mode presents the user with a panoramic acoustic image of the environment. A sweep of stereophonic sounds appears to "travel" through the user's head from the right to the left ear. The direction to an object is indicated by the spatial direction of the signal, and the distance is represented by the signal's pitch and volume (higher pitch and volume for shorter distances). As the information in this mode is more detailed than the information in the Guidance Mode, subjects found it more difficult to interpret the signals and react to them quickly. To reduce the amount of information transferred, unnecessary information is suppressed and only the most important sections of the environment are transmitted to the user. The selection of these relevant sections from the panoramic map is performed by the computer based on proximity of objects in the direction of travel. For example, when traveling in a crowded street or when entering a narrow passage, the computer transmits information about the sectors containing the closest objects to the user while ignoring all other, more distant objects.

3) Directional Guidance Mode - This mode allows the user to control the global navigation while the obstacle avoidance is performed by the NavBelt. The system actively guides the user toward a temporary target, the location of which is determined by the user via a joystick. The joystick used for this purpose during the development of the Navbelt may not be ideal for this purpose in a final product. A special auditory coding system or a speech control device may conceivably be better suited. The target position is selected according to the direction the joystick is pointing to. When the joystick is not pushed, the system selects a default target five meters ahead of the user. If the traveler wishes to turn sideways, he or she deflects the joystick in the desired direction and a momentary target is selected five meters ahead of the user in that direction. In case an obstacle is detected, the NavBelt provides the user with the relevant information to avoid the obstacle with minimal deviation from the target direction.

The variety of operational modes allows for different levels of assistance to the user, and different information formats. The Guidance mode is the most "automated" mode as the majority of the perception and cognition tasks are performed by the computer. This mode is efficient when the user is traveling in an unknown cluttered environment and the NavBelt serves as the primary aid. In the Image mode the computer tasks are limited to scanning the surroundings and informing the user about the position of obstacles, while the global path planning and navigation tasks are performed by the user.

The NavBelt's acoustic imaging technique can produce several informative parameters: 
- Direction of the audio signal's source: In the Image mode the signal produces a virtual source that represents the direction of the object. In the Guidance and Directional Guidance modes the virtual sound source represents the recommended travel direction.

- Volume: This parameter represents the proximity of the object to the user (Image mode) or the recommended traveling speed (Guidance modes).

- Pitch: In the Guidance modes the pitch is proportional to the complexity of the travel in that direction. This complexity depends on the distance to the nearest obstacle, number of obstacles, and the width between them (i.e. traveling through a narrow passage or among several small objects is more demanding than traveling in an uncluttered environment).

- Transmission rate: The signals' transmission rate is proportional to the conscious effort required from the user. When the NavBelt detects a potential hazard (a nearby obstacle, for example), the frequency at which the signals are transmitted (in all operation modes) is increased, thereby alerting the traveler.

The use of stereophonic displays in travel aid for the blind has already been implemented (i.e. the Sonicguide, [Kay, 1974]), and many researches deal with the problem known as auditory localization ([Vinje and Pitkin, 1971], [Ericson and Mckinley, 1989], [Wenzel, 1990]). However there are two major differences between the use of auditory localization in the Navbelt and the Sonicguide:

1. In the Guidance mode, the auditory cue signals the recommended travel direction, rather the location of an obstacle.

2. In the Image mode, the stereophonic sweep provides a full panoramic virtual image of the surrounding, thanks to the 120-degree wide coverage by the array of sensors. Furthermore, when traveling in a cluttered environment the sensors can detect several objects simultaneously, providing sufficient information for traveling through doorways, narrow passages etc.

\subsection{Implementation of the Guidance Mode}

In the guidance mode the computer provides the user only with the recommended travel speed and direction, based on the VFH obstacle avoidance algorithm (see Section 3.2.1). The VFH method calculates the travel direction from the polar histogram by searching for sectors with a low so-called "obstacle density" value. In practice, the VFH determines a threshold level, and all sectors with lower obstacle density than that level become candidate sections. Next, the VFH searches for the candidate sector that is closest to the direction of the target. The travel speed is determined by the VFH method according to the proximity of the robot (or human in the Navbelt) to the nearest object. The speed is determined inversely proportional to the minimal distance, with maximum speed of $1.2 \mathrm{~m} / \mathrm{sec}$ attained when the distance between the traveler and the closest object is larger than 3 meters.

The recommended travel speed and direction are relayed to the user by a single stereophonic signal. The virtual direction of the signal is the direction the obstacle avoidance system has selected for travel. The pitch and amplitude are proportional to the recommended travel speed. Higher pitch and amplitude attract more human attention [Benson, 1986], thereby motivating the traveler to reduce the walking speed and to concentrate on the stereophonic signal. A special low pitch signal $(250 \mathrm{~Hz})$ is transmitted when the direction of motion coincides (within $\pm 5^{\circ}$ ) with the required direction. This special tone is a simple feedback signal for the user, indicating that the travel direction is correct. Furthermore, low 
pitch tones occlude external sound from the environment less than medium and high pitch tones do [Benson, 1986]. The higher pitch tone is transmitted only when the traveler needs to change the travel direction, and as soon as that direction coincides with the recommended direction the low pitch returns.

Another important parameter involved in the guidance mode is the rate at which signals are transmitted. Although a low transmission rate causes less occlusion of external sounds, it may also be too slow to alert the traveler to hazards. An adaptive information transfer system adjusts the transmission rate according to changes in the process and the user's requirements, similar to the way the information flow is adjusted in the Image mode. When the user is traveling in an unfamiliar environment cluttered with a large number of obstacles, the transmission rate increases, and may reach up to 10 signals per second. On the other hand, when traveling in an environment with little or no obstacles, the transmission rate is reduced to one signal every three seconds.

\subsection{Implementation of the Image Mode}

As previously mentioned, the image mode provides the user with a panoramic auditory image of the surroundings. The principle is similar to the operation of radar systems used in air traffic control, submarines, etc. An imaginary beam travels from the right side of the user to the left through the sectors covered by the NavBelt's sonars (a range of $120^{\circ}$ and $5 \mathrm{~m}$ radius). A binaural feedback system invokes the impression of a virtual sound source moving with the beam from the right to the left ear in what we call a sweep. This is done in several discrete steps, corresponding to the discrete virtual direction steps. The angular displacement of the virtual sound source is obtained by a combination of the interaural phase and amplitude shift of the left and right signals. The phase shift is based on the different perception time of an auditory signal due to the difference travel distance of the sound wave. The phase shift ( $i \times N$ terms of time difference between left and right ears) is given by Eq. 1:

$$
\Delta t=K \cos \theta
$$

where $K=0.000666 \mathrm{sec}$ is the time phase constant, and $\theta$ is the angular position of the virtual source from the median plane in front of the user (see Figure 7). The interaural amplitude difference of a sound source due to angular shift is given by:

$$
\theta=K \log \left[\frac{A_{R}}{A_{L}}\right]+\theta_{0}
$$

where $A_{R}$ and $A_{L}$ are the amplitudes to the right and

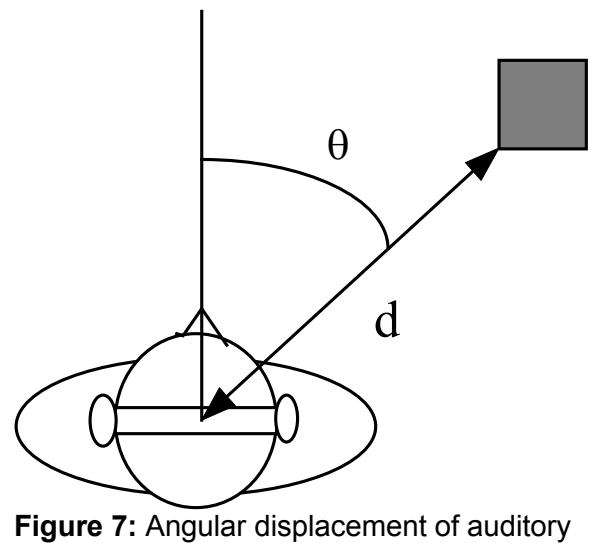
source

left ears, $K$ is the sensitivity factor and $\theta_{0}$ is a constant offset. Rowel [1970] showed that for most of the range of audible frequencies the sensitivity constant is 2 . For the NavBelt we therefore assume $K=2$ and $\theta_{0}=0$. The amplitude of the dominant channel - the channel closest to the object - is set according to the proximity to the object in that direction. For example for the configuration shown in Figure 7 the amplitude of right channel, $A_{R}$, is set proportionally to the distance $d$ and the amplitude of the left channel is therefore:

$$
A_{L}=\frac{1}{A_{R}} e^{\theta_{n}}
$$


At each step, the amplitude of the signal is set proportionally to the distance to the object in that virtual direction. If no obstacles are detected by the beam, the virtual sound source is of a low amplitude and barely audible. If, on the other hand, obstacles are present, then the amplitude of the virtual sound source is louder.

Figure 8 demonstrates the principle of the image mode. Obstacles are detected by the ultrasonic sensors (Figure 8a), and are projected onto a so-called polar histogram ${ }^{2}$, as shown in Figure 8b. Based on the polar histogram, the binaural feedback system generates the sweep, which comprises of 12 steps (Figure 8c). Each step "covers" a sector of $15^{\circ}$, so that the whole sweep covers a panorama of $180^{\circ}$. Each of the eight sectors in the center of the panorama (covering the sectors between $30^{\circ}$ and $150^{\circ}$ ) is directly proportional to the corresponding sensor. The remaining four sectors (two at each side of the panorama) represent sectors which are not covered by the sonars. The value of these sectors is extrapolated based on the averaged values of adjoining sectors. For example, if the third and forth sector (representing the first and second sonar) contain an object, than the first and second sectors are automatically assigned the averaged value.

Each signal is modulated by an amplitude $A$ (indicating the distance to the obstacle in that direction), the duration $T_{S}$, for which the square wave signal is audible, and the pitch $f$ of the square wave. The spacing time $T_{n}$ is the length of the interval between consecutive signals during a sweep. After each sweep there is a pause of duration $T_{\mathcal{C}}$, to allow the user to comprehend the conveyed image. Many meaningful combinations of these parameters are possible. For example, because of the short-term memory capability of the human ear, a sweep may be as short as 0.5 sec. Given enough cognition time $T_{c}$, the user will comprehend the image. Alternatively, the sweep time may be as long as one second, combined with a very short cognition time. Notice that

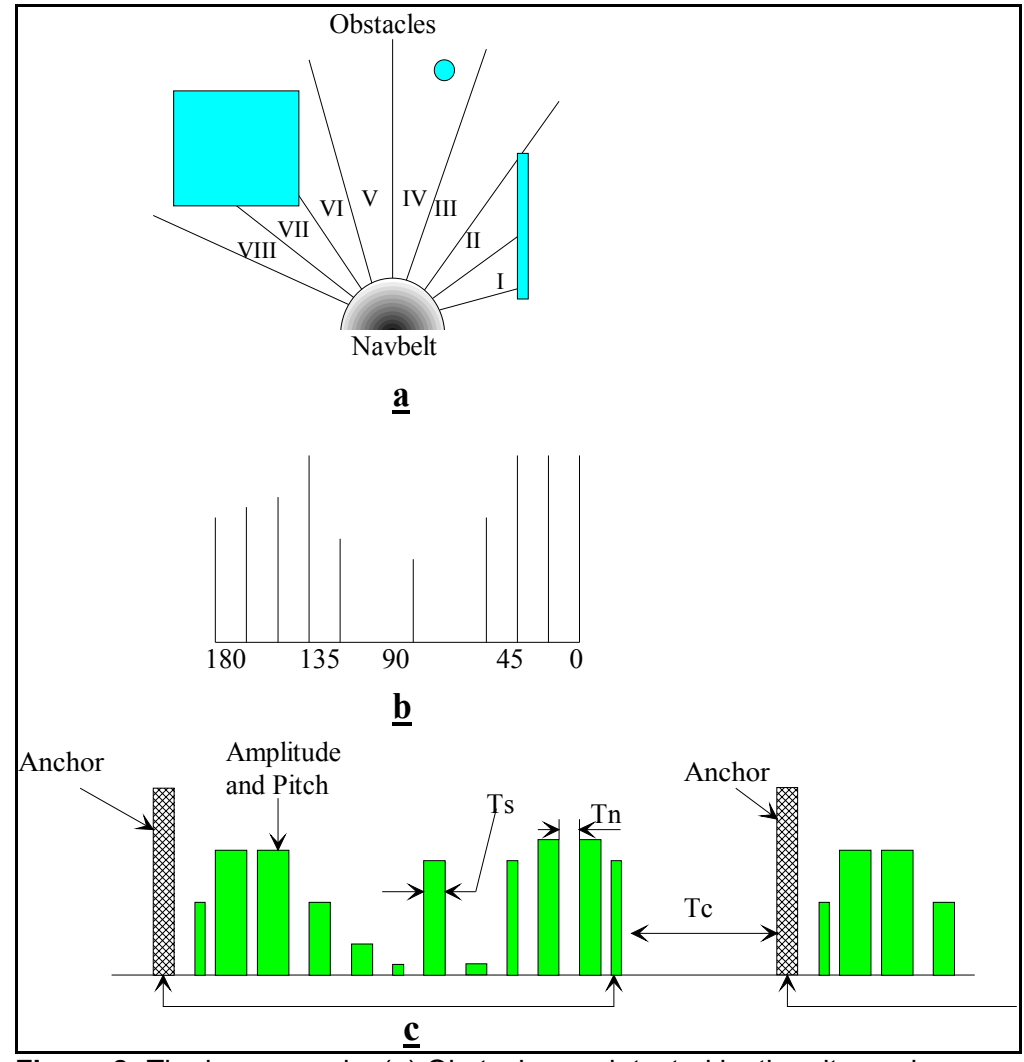

Figure 8: The image mode. (a) Obstacle are detected by the ultrasonic sensors. (b) Sonar range readings are projected onto the polar histogram. (c) An acoustic sweep is generated from the polar histogram.

2 How this is done is explained in greater detail in Section 5.5. 
each sweep starts with an anchor signal. This signal has a unique pitch, which provides the user with a convenient marker of the start of a sweep.

One of the important features of the image mode is the acoustic directional intensity (ADI), which is directly derived from the polar histogram. The virtual direction of the ADI provides information about the source of the auditory signal in space, indicating the location of an object. The intensity of the signals is proportional to the size of the object and its distance from the person as directly derived from the polar histogram.

The directional intensity is a combination of the signal duration $T_{S}$, the amplitude $A$, and the pitch. Experiments with human auditory perception show [Benson, 1986] that the perceived intensity increases with the signal's amplitude, pitch, and duration. Adjusting the acoustic directional intensity according to the location of obstacles in the surroundings attracts the user's attention to the most relevant sections in the environment, while suppressing irrelevant data.

The information adjustment is based on updating the sweep intensity according to the human and environment models. For example, if the human reaction is not satisfactory, than the sweep transmission rate and the ADI are increased. Similarly, the transmission rate and intensity are reduced when the expected performance (calculated from the user and environment models) shows that few obstacles are ahead.

\subsection{Experimental Results}

The NavBelt was extensively tested during its five-year long development. Some of the key experimental results are presented in the following section.

\subsubsection{Experiments with Real Obstacles}

In this experiment subjects walked through laboratory obstacle courses comprising various types of obstacles and using the various operation modes of the NavBelt. In the first experiment several vertical poles with different diameters were positioned along the travel path. It was found that the NavBelt can detect objects as narrow as $10 \mathrm{~mm}$. However, this can be done only if the objects are stationary and the subject is walking slowly (less than $0.4 \mathrm{~m} / \mathrm{sec}$ ). It was also found that the NavBelt can reliably detect objects with a diameter of $10 \mathrm{~cm}$ or bigger, regardless of the travel speed. Other tests were conducted inside office building where subjects traveled along corridors, located doors and curves, and detected and avoided furniture.

In other experiments subjects traveled outside buildings, detecting and avoiding common objects such as trees and large bushes, parked cars, walls, bicycles, and other pedestrians. One major concern of users was the lacking ability of the current prototype NavBelt to detect overhanging objects, up- and down-steps, sidewalk edges, etc. Future improvements to the NavBelt will require the addition of sonars pointing up and down to detect these type of obstacles.

\subsubsection{Experiments with Different Walking Patterns}

The next experiment tested the NavBelt in terms of walking patterns. It was found that uneven walking patterns cause the sonars to move along the vertical plane (sonars swinging up and down), which reduce the reliability of the sonar data. In addition, it was found that the relative angle between the sonars and the vertical orientation of the NavBelt (the angle of the 
sonars with the horizon) affects object detection. For example, if the NavBelt is tilted by $\pm 5^{\circ}$ from the horizon, the sonar reading can be off by more than $9 \%$. Swinging the arms during normal walking pattern did not interfere with the sonar performance as no sonars are directed to the sides. However, using the White cane (the most common device used by blind travelers) can cause interference to the sonar performance, mainly when it is used to detect objects above the ground level (higher than $0.5 \mathrm{~m}$ ). However, since the cane is used mainly to detect objects at ground level, while the NavBelt is designed to detect objects above ground level, this interference is not critical to the general performance.

The experiments with the NavBelt prototype showed the importance of training. Subjects with more experience traveled faster and generally were more comfortable. After 20 hours of practice with a NavBelt simulator and 40 hours practice with the experimental prototype NavBelt subject traveled at $0.8 \mathrm{~m} / \mathrm{sec}$ in the Guidance mode and $0.4 \mathrm{~m} / \mathrm{sec}$ in the Image mode. Subjects with less experience (10 hours with the simulator and 10 hours with the prototype) traveled at an average speed of $0.6 \mathrm{~m} / \mathrm{sec}$ in the Guidance mode and $0.3 \mathrm{~m} / \mathrm{sec}$ in the Image mode.

\subsection{Conclusions}

In this section we discussed the concept, implementation, and experimental results of the NavBelt, a portable navigation aid for the blind. The NavBelt offers three modes of operation: (1) Guidance Mode, (2) Directional Guidance Mode, and (3) Image Mode.

Both Guidance modes were found to be effective, allowing reasonably high walking speeds after a relatively small amount of practice (on the order of $40+$ hours). The problem with both Guidance modes, however, is the fact that they require accurate feedback about the user's momentary position and heading. With the current state of technology, this feedback is not available, and the two Guidance modes can thus not be implemented for practical purposes.

The Image mode provides the user with more detailed information about obstacles in the environment - a feature found to be desirable by some of the blind subjects that were interviewed in the course of the development. However, the "cost" of the increased information is that it takes substantially longer to comprehend the acoustic images provided by the NavBelt. It also took substantially longer to become practiced in the interpretation of the acoustic images. After 100 hours of practice in the Image mode a user could walk through a moderately dense obstacle course at walking speeds of about $0.3-0.4 \mathrm{~m} / \mathrm{sec}$.

We concluded that the Image mode is too slow to be practical for most users, although we believe that performance would further improve with much additional practice. We also concluded that advances in position estimation technology for pedestrians might make the NavBelt's Guidance modes more practical. Some recent developments have been made in this direction and resulted in a commercially availability device called PointMan ${ }^{\mathrm{TM}}$ Navigator II, which combines GPS and personal dead reckoning [Point Research]. However, we haven't tested this device at our lab. 


\section{The GuideCane}

The foremost problem with the NavBelt, as concluded above, are the difficulty of conveying information (in Image mode) to the user to allow him/her to react in time to obstacles ahead. Even if the NavBelt's Guidance modes could be implemented in practice, they would still require the user to concentrate on the acoustic guidance signals and to react to them quickly and efficiently.

A new invention made at the University of Michigan's Mobile Robotics Lab in 1995 aimed at overcoming these problems. This invention, called GuideCane, can be thought of as a robotic guide-dog, but it does not have the disadvantages of mobile robot guide-dogs discussed in Section 3.2.2).

Figure 9 shows a schematic view of the GuideCane and its functional components. Much like the widely used white cane, a user holds the GuideCane in front of himself/herself while walking. The GuideCane is considerably heavier than the white cane, but it rolls on wheels that support the GuideCane's weight during regular operation. A servomotor, operating under the control of the GuideCane's built-in computer, can steer the wheels left and right relative to the cane. Both wheels are equipped with encoders to determine their relative motion. For obstacle-detection, the GuideCane is equipped with ten ultrasonic sensors. To specify a desired direction of motion, the user operates a mini joystick located at the handle. Based on the user input and the sensor data from its sonars and encoders, the built-in computer decides where to head next and turns the wheels accordingly. Similar to the NavBelt, the GuideCane is equipped with an array of ultrasonic sensors and an obstacle avoidance system.

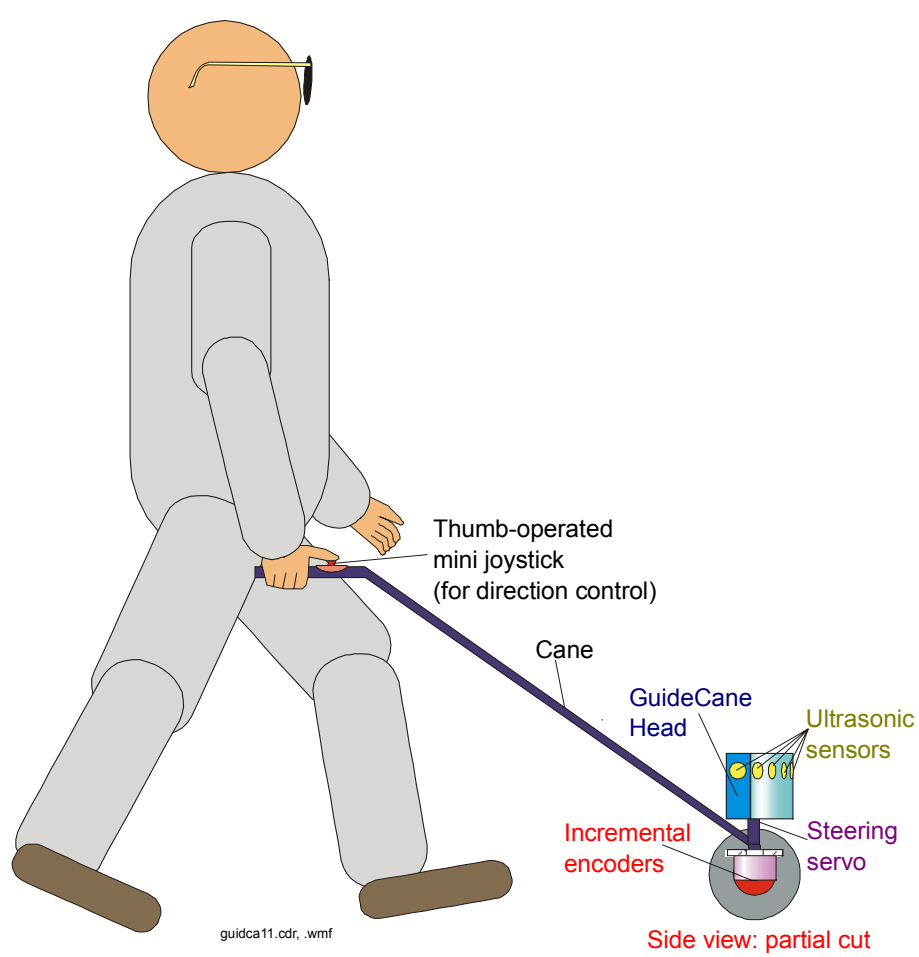

Figure 9: Functional components of the GuideCane.

\subsection{Functional Description}

During operation, the user pushes the GuideCane forward. Using the thumb-operated mini joystick, the user can prescribe a desired direction of motion. This directional command is understood to be relative to the GuideCane's current direction of motion. For example, if the user presses the button forward, then the system considers the current direction of travel to be the desired direction. If the user presses the button to the left, then the computer adds $90^{\circ}$ to the current direction of travel and, as soon as this direction is free of obstacles, steers the wheels to the left until the $90^{\circ}$ left turn is completed. It is important to note 
that the user can usually indicate a new direction well before the change of direction should occur. In the case of a corridor, if the user presses the button to the left, then the GuideCane will continue down the corridor until it reaches an intersection where it can turn to the left. The ability to indicate a desired direction of motion in advance significantly enhances the GuideCane's ease-of-use.

While traveling, the ultrasonic sensors detect any obstacle in a $120^{\circ}$ wide sector ahead of the user (see Step 1 in Figure 10). The built-in computer uses the sensor data to instantaneously determine an appropriate direction of travel, even among densely cluttered obstacles. If an obstacle blocks the desired travel direction, then the obstacle avoidance algorithm prescribes an alternative direction that clears the obstacle and then resumes in the desired direction (see Step 2 in Figure 10).

Once the wheels begin to steer sideways to avoid the obstacle, the user feels the resulting horizontal rotation of the cane (see Step 3 in Figure 10). In a fully intuitive response, requiring virtually no training time, the traveler changes his/her orientation to align himself/herself with the cane at the "nominal" angle. In practice, the user's walking trajectory follows the trajectory of the GuideCane similar to the way a trailer follows a truck. Because of the handle's short length, the user's trajectory is very close to the GuideCane's trajectory. Once the obstacle is cleared, the wheels steer back to the original desired direction of travel. The new line of travel will be offset from the original line of travel. Depending on the circumstances, the traveler may wish to continue walking along this new line of travel, or the system can be programmed to return to the original line of travel. This latter option is made possible by the GuideCane's dead-reckoning capability.

A particular problem is the detection of stairs. The GuideCane offers separate solutions for downsteps and up-steps. Down-steps are detected in a failsafe manner: when a down-step is encountered, the wheels of the GuideCane drop off the edge until the shock-absorbing bottom hits the step - without a doubt a signal that the user can not miss. Because the user walks behind the GuideCane, he/she has sufficient time to stop. Up-steps can be detected by additional front-facing sonars as described in [Borenstein and Ulrich, 1997]; however, this method is not yet implemented in the GuideCane.

\subsection{Guidance Signals versus Obstacle Information}

Existing ETAs are designed to notify the user of obstacles, usually requiring the $\mathrm{him} / \mathrm{her}$ to perform some sort of scanning action. The user must evaluate all of the obstacle information, which comprises of the size and proximity of each obstacle and then decide on a suitable travel direction. In sighted people, such relatively high bandwidth information is processed almost reflexively, usually without the need for conscious decisions. Nature had millions of years

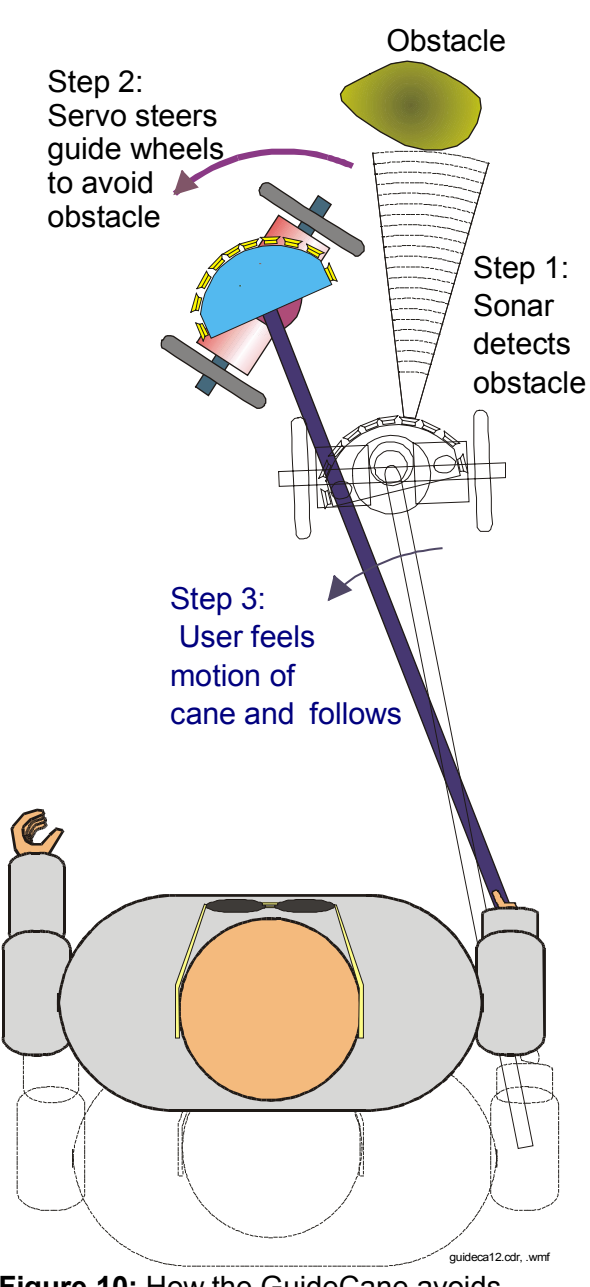

Figure 10: How the GuideCane avoids obstacles. 
of evolution to perfect this skill. However, the evaluation of obstacle information presented acoustically is a new skill that must be acquired over hundreds of hours of learning, as we concluded in Section 4.5 and in [Shoval et al., 1998b]. Even then, exercising such a skill requires a great deal of conscious effort, and thus processing time. The required effort further increases with the number of detected obstacles.

The GuideCane is fundamentally different from other devices in that it first analyzes the environment and then computes the momentary optimal direction of travel. The resulting guidance signal is a single piece of information - a direction - which means that the bandwidth of the information is much smaller. The consequence is that it is far easier and safer to follow the low-bandwidth guidance signal of the GuideCane than to follow the highbandwidth information of other existing systems. However, reducing the high-bandwidth obstacle information to a momentary optimal direction of travel requires the use of advanced mobile robot technologies.

\subsection{Information Transfer}

In prior research with the NavBelt, different methods were tested that use binaural (stereophonic) signals to guide the user around obstacles as described in Section 4. Subjects found it difficult to recognize and react to such signals at walking speed [Shoval et al., 1998b]. By contrast, our tests have shown that untrained subjects could immediately follow the GuideCane at walking speed, even among densely cluttered obstacles.

This advantage can be credited to another unique feature of the GuideCane: information transfer through direct physical force. This process is completely intuitive so that everybody can use the system right away without learning how to interpret artificially defined acoustic or tactile signals, as with existing ETAs. Yielding to external forces is a reflexive process that does not require a conscious effort. Moreover, many blind persons are accustomed to being guided by sighted people in a similar fashion.

Even though the GuideCane's wheels are unpowered, the GuideCane can apply a substantial amount of physical force on the user. The sideways motion of the wheels results in a rotation of the handle of the cane, which is clearly noticeable. A second force, immediately felt after the wheels change their orientation (but even before the user feels the rotation of the cane), is the increased reaction force that is opposed to pushing the cane forward. When walking while the cane and the wheels are aligned, the user must only overcome the reactive force $F_{r}$ resulting from the friction in the bearings and the roll resistance of the wheels. If the wheels steer an angle $\theta$ in either direction of the cane, then the user has to push the cane with an increased force equal to $F_{r} / \cos \theta$ to overcome the reactive force of the wheels. This change in reactive force is immediately felt by the user and prepares $\mathrm{him} / \mathrm{her}$ for an upcoming steering maneuver.

\subsection{Hardware Implementation}

The GuideCane must be as compact and as lightweight as possible so that the user can easily lift it, e.g., for coping with stairs and access to public transportation. For the same reason, the electronic components should require minimal power in order to minimize the weight of the batteries. In addition, both the mechanical and electronic hardware must be designed to facilitate the software's task: allowing real-time performance with limited onboard processing power. 


\subsubsection{Mechanical Hardware}

The GuideCane consists of three main modules: housing, wheelbase, and handle. The housing contains and protects most of the electronic components. The current prototype is equipped with ten Polaroid ultrasonic sensors that are located around the housing. Eight of the sonars are located in the front in a semi-circular fashion with an angular spacing of $15^{\circ}$, covering the area ahead of the GuideCane with a total angular spacing of $120^{\circ}$. The other two sonars face the sides and are particularly useful for following walls and for going through narrow openings, such as doorways.

The wheelbase can be rotated by a small servomotor. As shown in Figure 11, the wheelbase uses ball bearings to support two unpowered wheels. To perform odometry, both wheels are equipped with lightweight quadrature encoders. Using full quadrature decoding, the resolution of the encoders is 2,000 pulses per revolution, resulting in more than 5 pulses for a wheel advancement of $1 \mathrm{~mm}$. The GuideCane's odometry equations are the same as for a differential drive mobile robot. However, because the wheels are unpowered, there is considerably less risk of wheel slippage.

The handle serves as the main physical interface between the user and the GuideCane. The angle of the handle can be adjusted to accommodate users of different heights. At the level of the user's hand, a joystick-like pointing device is fixed to the handle. The pointer consists of a mouse button (similar to the pointing devices used on some notebook computers) that the user can press with his/her thumb in any direction. The selected direction indicates the desired direction of travel relative to the current orientation of the cane. In the current implementation, this direction is discretized into eight directions.

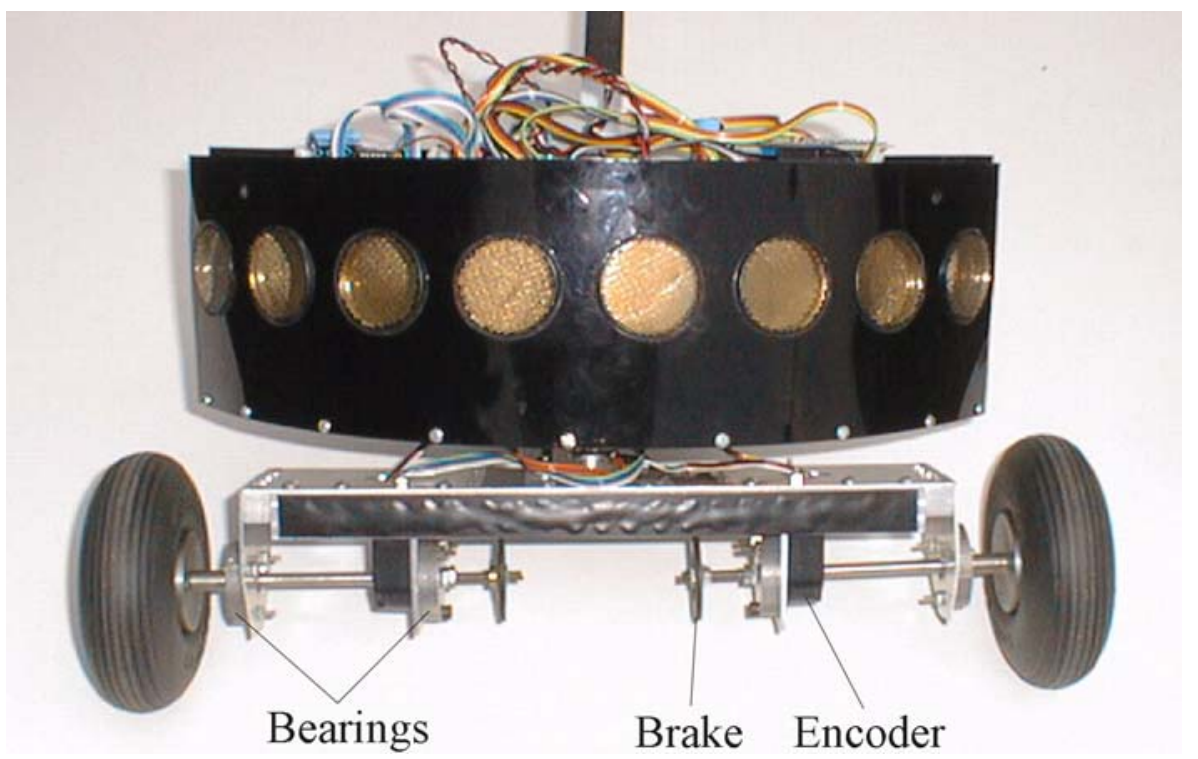

Figure 11: The GuideCane housing and wheelbase. 


\subsubsection{Electronic Hardware}

The electronic system architecture of the GuideCane is shown in Figure 12. The main brain of the GuideCane is an embedded PC/104 computer, equipped with a 486 microprocessor that is clocked at $33 \mathrm{MHz}$. The PC/104 stack consists of four layers. Three of the modules are commercially available boards, including the motherboard, the VGA utility module, and a miniature 125-MB harddisk. The fourth board, which was custom-built, serves as the main interface between the PC and the sensors (encoders, sonars, and potentiometer) and actuators (main servo and brakes). The main interface executes many time-critical tasks, such as firing the sonars at specific times, constantly checking the sonars for an echo, generating the PWM signals for the servos, and decoding the encoder outputs. The main interface also acts as an asynchronous buffer for the sonar data. Although the GuideCane currently uses only ten sonars, the main interface provides hardware and software support for up to 16 sonars.

The PC is connected to the main interface through its bi-directional parallel port. The interface preprocesses most of the sensor data before the data is read by the PC. In addition, all communications are buffered. The preprocessing and buffering not only minimizes the communications between the $\mathrm{PC}$ and the interface, but also minimizes the computational burden on the PC to control the sensors and actuators. Because the main interface completes all the low-level tasks, almost all of the PC's computational power can be dedicated to medium and high-level tasks. The interface consists of three MC68HC11E2 microcontrollers, two quadrature decoders, a FIFO buffer, and a decoder. Thus, the GuideCane can be described as an embedded system equipped with four processors.

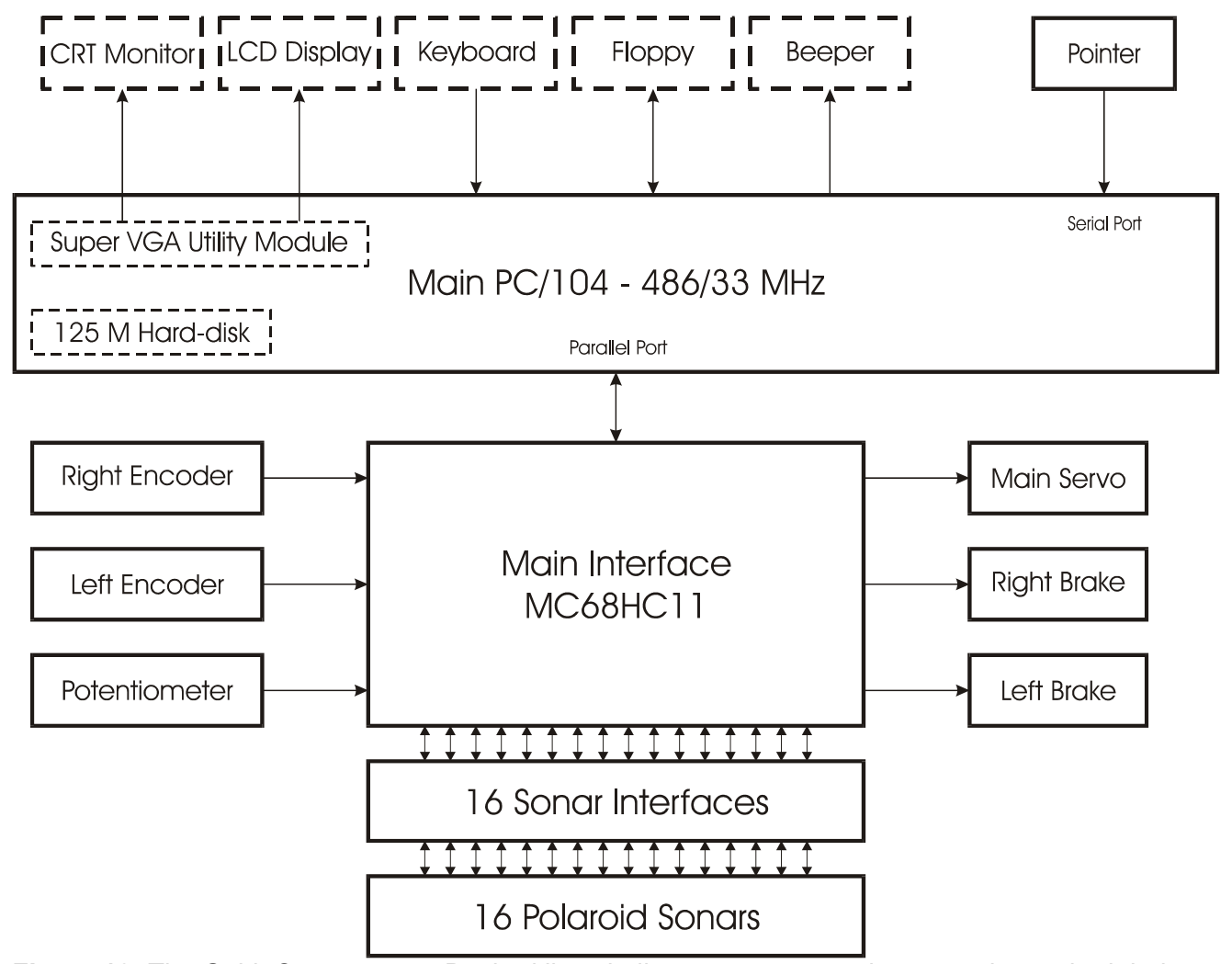

Figure 12: The GuideCane system. Dashed lines indicate components that are only required during the development stage. 
The embedded PC/104 computer provides a convenient development environment. For stationary development, the system is connected to a regular keyboard and a CRT monitor. For mobile tests, the computer is connected to a smaller keyboard and a color LCD screen that is fixed to the handle a little below the developer's hand. The entire system is powered by rechargeable NiMH batteries, allowing mobile testing for several hours. Even during development the GuideCane is a truly autonomous system in the sense of power and computational resources.

Even though the current prototype consists of four PC/104-sized modules, only two of them are required during operation. One module that provides the VGA display interface is very useful for visual feedback during development, but it is not needed during operation. Another module that houses the hard-disk can also be eliminated in a final product. The final software will be stored in an EPROM that can be added to the motherboard. This solid-state solution will also eliminate potential problems with the moving parts of the hard-disk, which is sensitive to shocks and vibrations. All components that can be eliminated in a final product version of the GuideCane are indicated in Figure 12 by boxes drawn with dashed lines.

\subsection{Software Implementation}

The GuideCane is a semi-autonomous system, providing full autonomy for local navigation (obstacle avoidance), but relying on the skills of the user for global navigation (path planning and localization). Combining the skills of a mobile robot with the existing skills of a visually impaired user is the key idea behind the NavBelt and the GuideCane. This combination of skills is what makes this particular application feasible at the current stage of mobile robotics research. While reliable global navigation algorithms might be available in the future, they are not essential for the GuideCane. Although visually impaired people have difficulties performing fast local navigation without a travel aid, they are in most cases perfectly capable of performing global navigation.

The main task of the GuideCane is to steer around obstacles and to proceed toward the desired direction of travel. The GuideCane's performance is thus directly related to the performance of its obstacle avoidance algorithm. To achieve safe traveling at fast walking speeds through cluttered and unknown environments, the GuideCane employs mobile robot obstacle avoidance methods that were developed earlier at the University of Michigan's Mobile Robotics Lab.

\subsubsection{Local Map Building}

Like the NavBelt, the GuideCane also uses EERUF to control the ultrasonic sensors to achieve a fast firing rate [Borenstein and Koren, 1995]. Each of the ten sonars is fired at a rate of $10 \mathrm{~Hz}$, so that the GuideCane receives 100 sonar readings per second. EERUF's fast firing rate is a key factor for the reliability and robustness of the GuideCane's obstacle avoidance performance and is necessary for allowing safe travel at fast walking speeds. And, also as in the NavBelt, the GuideCane employs the VFH obstacle avoidance method described in Section 3.2.1. However, several improvement over the original VFH method were implemented in the GuideCane. The improved method, called VFH+, is discussed next.

One of the improvements of VFH+ is that the polar histogram is modified to rule out those obstacle-free directions that cannot be taken because of the kinematic and dynamic constraints of the GuideCane (see [Ulrich and Borenstein, 1998] for details). The modified polar histogram is called the masked polar histogram. A threshold applied to the masked 
polar histogram determines which directions are actual candidates for travel. A hysteresis is applied to the threshold to provide smoother and less oscillatory motion.

The VFH+ algorithm selects the most suitable direction (out of all candidate directions) of motion based on a so-called cost function. The cost function includes three terms: the angular deviation from the desired direction, the angular deviation from the current orientation of the wheelbase, and the angular deviation from the previously selected direction of motion. For each candidate direction the cost of choosing this direction is computed, using the fixed, pre-programmed cost function. Selecting the most suitable direction therefore means simply selecting the candidate direction with the lowest cost.

Although the performance of the $\mathrm{VFH}+$ method is adequate in most situations, it sometimes directs the GuideCane into local dead-ends (e.g., concave obstacles) that were detected as such by its sensors, and thus could have been avoided. To avoid steering into such dead-ends, the VFH+ method was combined with the $A^{*}$ search algorithm, and includes an appropriate cost, a heuristic function, and a discounting factor [Ulrich, 1997]. The A* search algorithm is used to find an optimal path of a short length, using the VFH+ method to determine suitable directions of motion at a given position (state).

\subsection{Experimental Results}

The actual GuideCane prototype, shown in Figure 13, was extensively tested at the University of Michigan's Mobile Robotics Laboratory.

A performance analysis of the experimental GuideCane prototype can be divided into two

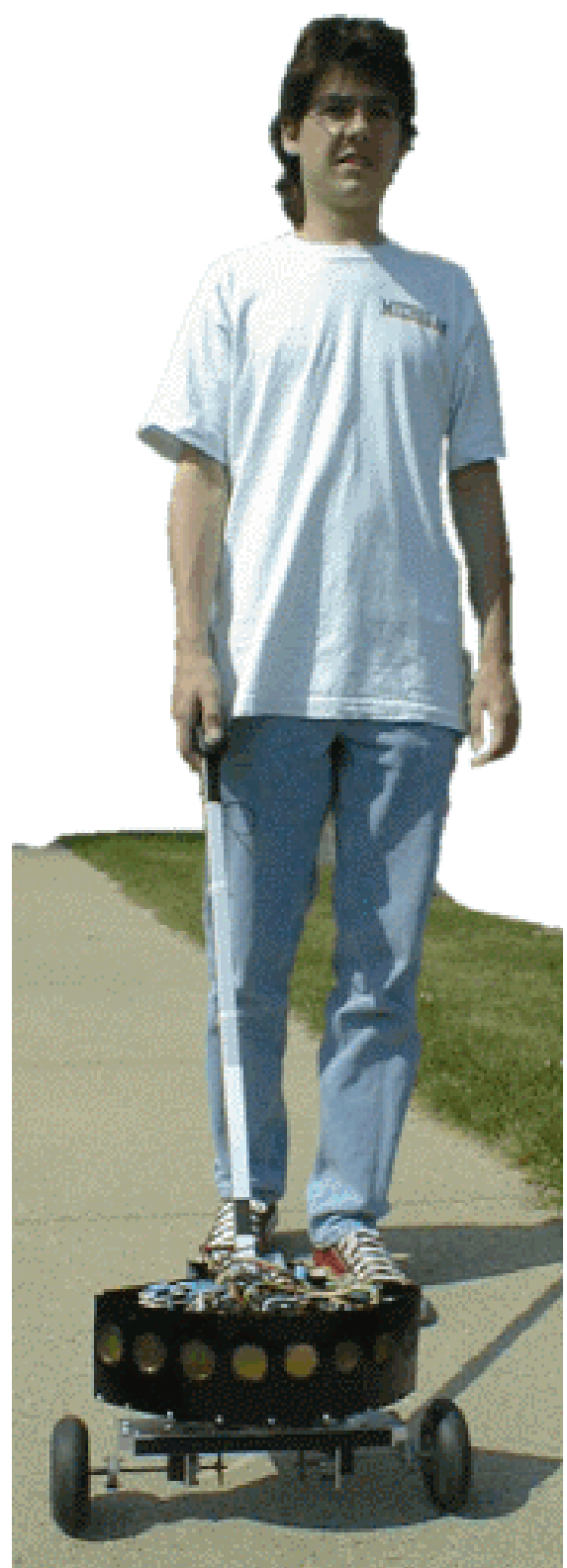

Figure 13: The actual GuideCane prototype.

categories: 1) the usefulness of the concept and 2) the performance of the obstacle avoidance system. The GuideCane concept fulfilled all our expectations and confirmed our initial hypothesis that following the GuideCane is a completely intuitive process. All subjects were able to follow the GuideCane easily at fast walking speeds of up to $1 \mathrm{~m} / \mathrm{sec}$, while completing complex maneuvers through cluttered environments. Subjects rarely needed more than a few minutes to get used to the GuideCane. Actually, blind subjects needed a few minutes to understand the GuideCane concept, as they could not visually observe how the device was working. Blindfolded subjects, on the other hand, needed some time to simply become accustomed to walking around without sight. Nonetheless, blind and blindfolded subjects alike observed that walking with the GuideCane was completely intuitive and did not require any conscious effort. 
The second category, the obstacle avoidance performance, is adequate in many indoor environments. The performance of the combined EERUF/HIMM/VFH+ system is excellent as long as the obstacles are indeed detected by the sonars. Screen captures of two test runs with the actual GuideCane are shown in Figure 14, demonstrating the processes of the local map building and the obstacle avoidance algorithms.

Failures of the obstacle avoidance system were in most cases caused by obstacles that were not detected by the sonars. For example, the GuideCane is currently not able to detect overhanging obstacles like tabletops. However, these obstacles should easily be detected with the additional upward-looking sonars of the next prototype version. The addition of these sonars is expected to improve the GuideCane's performance to a level where a visually impaired person could effectively use the device indoors. Outdoors, however, the implementation of an additional type of sensor will be required to allow the GuideCane to detect important features, such as sidewalk borders. In order for the GuideCane to become a truly useful tool for a visually impaired person, it will be essential to develop a real-time method for the detection of these features.



a

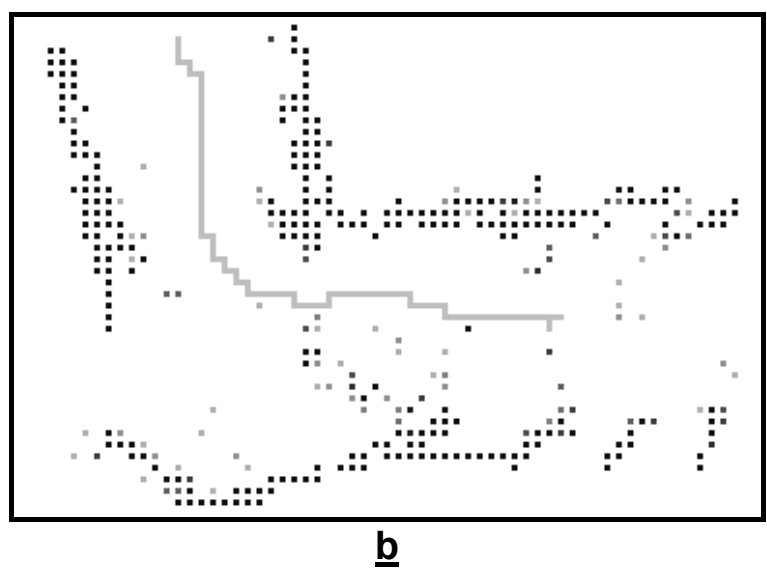

$\underline{\mathbf{b}}$

Figure 14: Two screen captures showing the path of the actual GuideCane through corridors. In both experiments the target direction is towards the right. The intensity of a histogram grid cell indicates its certainty value. The gray line indicates the robot's trajectory, based on its odometry.

a). The GuideCane continues down a corridor.

b) The GuideCane makes a left turn at a T-shaped intersection

\section{Discussion}

Both the NavBelt and the GuideCane successfully overcome the first two fundamental shortcomings of existing ETAs, as identified in Section 2. The first shortcoming is overcome by using multiple sensors that face in different directions, thus covering a large area regardless of the user's orientation. As a consequence, the user no longer needs to actively scan the surroundings manually.

The second shortcoming is overcome by the use of obstacle avoidance technology. Due to the multi-sensor system and the accumulation of sensor data, no additional measurements are required for the obstacle avoidance algorithm to determine a path that guides the blind 
traveler around the obstacles. In the case of the GuideCane, the obstacle avoidance algorithm provides full autonomy for local navigation, thus allowing the user to fully concentrate on the less time-constrained tasks of global path planning and localization. As a consequence, faster walking speeds can be achieved even with a smaller conscious effort on the user's part.

The NavBelt does not overcome the third shortcoming, as it relies on acoustic feedback and thus masks acoustic cues that are important for the user. The GuideCane successfully overcomes this shortcoming by using a much simpler and more direct interface that is based on physical force instead of acoustic feedback.

The GuideCane offers two additional advantages over the NavBelt. (1) the GuideCane is much more intuitive to use, and thus requires very little training time. (2) because the GuideCane rolls on wheels that are in contact with the ground, it can get an estimate of its position through odometry. The position estimation not only allows for the easier guidance mode, but it also allows for a better obstacle avoidance performance. On the other hand, the NavBelt could be used together with the white cane, which is not the case for the GuideCane.

\section{References}

1. Benjamin, J. M., Ali, N. A., and Schepis, A. F., 1973, "A Laser Cane for the Blind," Proceedings of the San Diego Biomedical Symposium, Vol. 12, pp. 53 - 57.

2. Benson K. B., 1986, "Audio Engineering Handbook," McGraw-Hill Book Company, New York.

3. Bissitt, D. and Heyes, A. D., 1980, "An Application of Biofeedback in the Rehabilitation of the Blind," Applied Ergonomics, Vol. 11, No. 1, pp. 31-33.

4. Blasch, B. B., Long, R. G., and Griffin-Shirley, N., 1989, "National Evaluation of Electronic Travel Aids for Blind and Visually Impaired Individuals: Implications for Design," RESNA $12^{\text {th }}$ Annual Conference, New Orleans, Louisiana, pp. 133-134.

5. Borenstein, J., and Koren, Y., 1991, "Histogramic In-Motion Mapping for Mobile Robot Obstacle Avoidance," IEEE Transactions on Robotics and Automation, August, pp. 535539.

6. Borenstein, J. and Koren, Y., 1991, "The Vector Field Histogram - Fast ObstacleAvoidance for Mobile Robots," IEEE Journal of Robotics and Automation, Vol. 7, No. 3., June, pp. 278-288.

7. Borenstein, J. and Koren, Y., 1995, "Error Eliminating Rapid Ultrasonic Firing for Mobile Robot Obstacle Avoidance," IEEE Transactions on Robotics and automation, February, Vol. 11, No. 1, pp. 132-138.

8. Borenstein, J., and Ulrich, I., 1997, "The GuideCane - A Computerized Travel Aid for the Active Guidance of Blind Pedestrians," IEEE International Conference on Robotics and Automation, Albuquerque, NM, April, pp. 1283-1288.

9. Brabyn, J. A., 1982, "New Developments in Mobility and Orientation Aids for the Blind," IEEE transaction on Biomedical Engineering, Vol. BAM-29, No. 4, pp. $285-$ 290.

10. Elfes, A., 1989, "Using occupancy grids for mobile robot perception and navigation," Computer Magazine, June, pp. 46-57. 
11. Ericson M. A., Mckinley R., L., 1989, "Auditory Localization Cue Synthesis and Human Performance," IEEE Proceedings of the National Aerospace and Electronics Conference, V2, pp. 718-725, NASEA9.

12. Fish R. M., "An Audio Display for the Blind," IEEE Transactions on Biomedical Engineering, Vol. BME-23, No. 2, March, pp. 144-153.

13. Golledge R. G., Loomis J. M. Klatzky R. L., Flury A., Yang X. L., 1991, "Designing a personal guidance system to aid navigation without sight: progress on GIS component," International Journal of Geographical Information Systems, Vol. 5, No. 4, pp. 373 -395.

14. Ifukube T., Sasaki T., Peng C., 1991, "A Blind Mobility Aid Modeled After Echolocation of Bats," IEEE Transactions on Biomedical Engineering, Vol. 38, No. 5, May, pp. 461-465.

15. Jackson, C., 1995, Correspondence with Carroll L. Jackson, Executive Director of the Upshaw Institute for the Blind, August 11, available at $\mathrm{ftp} / / / \mathrm{ftp}$. eecs.umich.edu/people/johannb/Carroll_Jackson_Letter.pdf.

16. Kay L., 1974, "A Sonar Aid to Enhance Spatial Perception of the Blind: Engineering Design and Evaluation," Radio and Electronic Engineer, Vol. 44, No. 11, pp. 605-627.

17. Kelly G. W., Ackerman T., 1982, "Sona, The Sonic Orientation and Navigational Aid for the Visually Impaired," Fifth Annual Conference on Rehabilitation Engineering, Houston, Texas, p. 72.

18. Lebedev, V. V. and Sheiman, V. L., 1980, "Assessment of the Possibilities of Building an Echo Locator for the Blind," Telecommunications and Radio Engineering, Vol. 3435, No. 3, pp. 97-100.

19. Mann R. W., 1974, "Technology and Human Rehabilitation: Prostheses for Sensory Rehabilitation and/or Sensory Substitution," Advances in Biomedical Engineering, Vol. 4, Academic Press, Inc. pp. 209-353.

20. Maure D. R., Mellor, C. M., Uslan M., 1979, "AFB's Computerized Travel Aid," Visual Impairment and Blindness, November issue, pp. 380-381.

21. Moravec, H. P., 1988, "Sensor fusion in certainty grids for mobile robots", AI Magazine, Summer, pp. 61-74.

22. POLAROID Corp, Ultrasonic Components Group, 119 Windsor Street, Cambridge, MA.

23. Point Research Corp., 2740 S. Harbor Blvd., Suite B, Santa Ana, California 92704, USA

714-557-6180,info@pointresearch.com, http://www.pointresearch.com/

24. Pressey N., 1977, "Mowat Sensor," Focus, Vol. 11, No. 3, pp. 35-39.

25. Rowel D., 1970, "Auditory Factors in the Design of a Binaural Sensory Aid for the Blind," Ph.D. Thesis, University of Canterbury, New Zealand.

26. Russell, L., 1965, "Travel Path Sounder," Proceedings of Rotterdam Mobility Res. Conference, New York: American Foundation for the Blind.

27. Shao, S., 1985, "Mobility Aids for the Blind," Electronic Devices for Rehabilitation, John Wiley \& Sons, New York, NY, pp. 79-100.

28. Shoval, S., Borenstein, J., and Koren, Y., 1994, "Mobile Robot Obstacle Avoidance in a Computerized Travel Aid for the Blind," IEEE International Conference on Robotics and Automation, San Diego, CA, May 8-13, pp. 2023-2029. 
29. Shoval, S., Borenstein, J., and Koren, Y., 1998, "The NavBelt - A Computerized Travel Aid For The Blind Based On Mobile Robotics Technology," IEEE Transactions on Biomedical Engineering, Vol. 45, No. 11, November, pp. 1376-1386.

30. Shoval, S., Borenstein, J., and Koren, Y., "Auditory Guidance with the NavBelt - A Computerized Travel Aid for the Blind," IEEE Transactions on Systems, Man, and Cybernetics, August 1998, Vol. 28, No. 3, pp. 459 - 467.

31. Tachi S., Mann R. W., Rowel D., 1983, "Quantitative Comparison of Alternative Sensory Displays for Mobility Aids for the Blind," IEEE Transactions on Biomedical Engineering, Vol. BMIE-30, No. 9, September, pp. 571-577.

32. Ulrich, I., and Borenstein, J., 1998, "VFH+: Reliable Obstacle Avoidance for Fast Mobile Robots", IEEE International Conference on Robotics and Automation, Leuven, Belgium, May, pp. 1572-1577.

33. Ulrich, I., 1997, "The GuideCane - A Computerized Travel Aid for the Active Guidance of Blind Pedestrians", Master Thesis, University of Michigan, MEAM, Ann Arbor, August.

34. Vinje, E. W., Pitkin E., T., 1971, "Human Operator Dynamics for Aural Compensatory Tracking," 7th Annual Conference on Manual Control, Los Angeles, California.

35. Wenzel E. M., 1990, "Localization in Virtual Acoustic Display," Human Interface Research Branch, Aerospace Human Factors Research Division, NASA Ames Research Center, MS-262, Presence: Vol. 1, No. 1.

36. Wormald International Sensory Aids, 6140 Horseshoe Bar Rd., Loomis, CA 95650. 